\title{
SOME RESULTS ON DOMINANT OPERATORS
}

\author{
YOUNGOH YANG \\ Department of Mathematics \\ Cheju National University \\ Cheju, 690-756, KOREA
}

(Received September 25, 1995 and in revised form January 30, 1997)

\begin{abstract}
We show that the Weyl spectrum of a dominant operator satisfies the spectral mapping theorem for analytic functions and then answer a question of Oberai.
\end{abstract}

KEY WORDS AND PHRASES: Fredholm, Weyl, dominant, $M$-power class $(N)$ 1991 AMS SUBJECT CLASSIFICATION CODES: 47A10, 47A53, 47B20,

\section{INTRODUCTION}

Throughout this paper $H$ will denote an infinite dimensional Hilbert space and $B(H)$ the space of all bounded linear operators on $H$. If $T \in B(H)$, we write $\sigma(T)$ for the spectrum of $T, \pi_{0}(T)$ for the set of eigenvalues of $T$, and $\pi_{00}(T)$ for the isolated points of $\sigma(T)$ that are eigenvalues of finite multiplicity. If $K$ is a subset of $\mathbb{C}$, we write iso $K$ for the set of isolated points of $K$. An operator $T \in B(H)$ is said to Fredholm if its range $\operatorname{ran} T$ is closed and both the null space $\operatorname{ker} T$ and $\operatorname{ker} T^{*}$ are finite dimensional. The index of a Fredholm operator $T$, denoted by $i(T)$, is defined by

$$
i(T)=\operatorname{dim} \operatorname{ker} T-\operatorname{dim} \operatorname{ker} T^{*} .
$$

The essential spectrum of $T$, denoted by $\sigma_{e}(T)$, is defined by

$$
\sigma_{e}(T)=\{\lambda \in \mathbb{C}: T-\lambda I \text { is not Fredholm }\} .
$$

A Fredholm operator of index zero is called a Weyl operator. The Weyl spectrum of $T$, denoted by $\omega(T)$, is defined by

$$
\omega(T)=\{\lambda \in \mathbb{C}: T-\lambda I \text { is not Weyl }\} .
$$

It was shown by Berberian [2] that $w(T)$ is a nonempty compact subset of $\sigma(T)$.

An operator $T \in B(H)$ is said to be dominant if for every $z \in \mathbb{C}$ there exists a real number $M_{z}>0$ such that

$$
(T-z)(T-z)^{*} \leq M_{z}(T-z)^{*}(T-z)
$$

In this case, if $\sup _{z \in \mathbb{C}} M_{z}=M<\infty, T$ is said to be $M$-hyponormal, and if $M=1, T$ is hyponormal. Evidently,

$$
T \text { is hyponormal } \Longrightarrow T \text { is } M \text {-hyponormal } \Longrightarrow T \text { is dominant }
$$

We also note that an operator $T$ need not be hyponormal even though $T$ and $T^{*}$ are both $M$-hyponormal. To see this, consider the operator

$$
T=\left[\begin{array}{cc}
U & K \\
0 & U^{*}
\end{array}\right]: l_{2} \oplus l_{2} \rightarrow l_{2} \oplus l_{2}
$$


where $U$ is the unilateral shift on $l_{2}$ and $K: l_{2} \rightarrow l_{2}$ is given by

$$
K\left(x_{1}, x_{2}, x_{3}, \cdots\right)=\left(2 x_{1}, 0,0, \cdots\right) .
$$

Then a direct calculation shows that

$$
\frac{1}{2}\|(T-z) x\| \leq\left\|(T-z)^{*} x\right\| \leq 2\|(T-z) x\|
$$

for all $z \in \mathbb{C}$ and for all $x \in l_{2} \oplus l_{2}$, which says that $T$ and $T^{*}$ are both dominant(even $M$-hyponormal). But since

$$
\left[\begin{array}{cc}
I & 0 \\
0 & I+\frac{3}{2} K
\end{array}\right]=T^{*} T \neq T T^{*}=\left[\begin{array}{cc}
I+\frac{3}{2} K & 0 \\
0 & I
\end{array}\right]
$$

$T$ is not normal(even hyponormal).

If $T$ is Fredholm then by (1.1)

$$
T \text { dominant } \Longrightarrow i(T) \leq 0 .
$$

It was known by Oberai [8] that the mapping $T \rightarrow \omega(T)$ is upper semi-continuous, but not continuous at $T$. However if $T_{n} \rightarrow T$ with $T_{n} T=T T_{n}$ for all $n \in \mathbb{N}$ then

$$
\lim \omega\left(T_{n}\right)=\omega(T) \text {. }
$$

It was known that $\omega(T)$ satisfies the one-way spectral mapping theorem for analytic functions: if $f$ is analytic on a neighborhood of $\sigma(T)$ then

$$
\omega(f(T)) \subset f(\omega(T)) .
$$

The inclusion (1.4) may be proper(see Berberian [2, Example 3.3]). If $T$ is normal then $\sigma_{e}(T)$ and $\omega(T)$ coincide. Thus if $T$ is normal since $f(T)$ is also normal, it follows that $\omega(T)$ satisfies the spectral mapping theorem for analytic functions. We say that Weyl's theorem holds for $T$ if

$$
\omega(T)=\sigma(T)-\pi_{00}(T) .
$$

It was known (Berberian [1]) that Weyl's theorem holds for any hyponormal operator - indeed, for any seminormal operator and for any Toeplitz operator. Oberai [9] has raised the following question: Does there exist a hyponormal operator $T$ such that Weyl's theorem does not hold for $T^{2}$ ? Note that $T^{2}$ may not be hyponormal even if $T$ is hyponormal(Halmos [5, Problem 209]).

In this paper we show that the Weyl spectrum of a dominant operator satisfies the spectral mapping theorem for analytic functions, and that Weyl's theorem holds for $p(T)$ when $T$ is hyponormal and $p$ is any polynomial. The latter result answers the question of Oberai.

\section{SPECTRAL MAPPING THEOREM}

THEOREM 2.1. If $S$ and $T$ are dominant operators, then

$$
S, T \text { Weyl } \Longleftrightarrow S T \text { Weyl. }
$$

PROOF. If $S, T$ are Weyl, then $S, T$ are Fredholm and $i(S)=i(T)=0$. By Conway [3], ST is Fredholm and by the index product theorem, $i(S T)=i(S)+i(T)=0$. Hence $S T$ is Weyl.

Conversely if $S T$ is Weyl, then $S T$ is Fredholm and $i(S T)=0$. Since $S$ and $T$ are dominant, $\operatorname{ker} S \subset \operatorname{ker} S^{*}$ and $\operatorname{ker} T \subset \operatorname{ker} T^{*}$. Since $\operatorname{ker} S^{*} \subseteq \operatorname{ker}(S T)^{*}, \operatorname{dim} \operatorname{ker} S \leq \operatorname{dim} \operatorname{ker} S^{*} \leq$ 
$\operatorname{dim} \operatorname{ker}(S T)^{*}<\infty$. Thus $\operatorname{ker} S$ and $\operatorname{ker} S^{*}$ are finite dimensional. By Schechter [10, Chap. 5 Theorem 3.5], $S$ and $T$ are Fredholm. Since $0=i(S T)=i(S)+i(T)$ by the index product theorem, by (1.2) $i(S)=i(T)=0$. Hence $S$ and $T$ are Weyl.

If the "dominant" condition is dropped in the above theorem, then the backward implication may fail even though $T_{1}$ and $T_{2}$ commute: For example, if $U$ is the unilateral shift on $l_{2}$, consider the following operators on $l_{2} \oplus l_{2}: T_{1}=U \oplus I$ and $T_{2}=I \oplus U^{*}$.

THEOREM 2.2. If $T$ is dominant and $f$ is analytic on a neighborhood of $\sigma(T)$, then $\omega(f(T))=f(\omega(T))$.

PROOF. Suppose that $p$ is any polynomial. Let

$$
P(T)-\lambda I=a_{0}\left(T-\mu_{1} I\right) \cdots\left(T-\mu_{n} I\right) .
$$

Since $T$ is dominant, $T-\mu_{2} I$ are dominant operators for each $i=1,2, \cdots, n$. It thus follows from Theorem 2.1 that

$$
\begin{aligned}
\lambda \notin \omega(p(T)) & \Longleftrightarrow p(T)-\lambda I=\text { Weyl } \\
& \Longleftrightarrow a_{0}\left(T-\mu_{1} I\right) \cdots\left(T-\mu_{n} I\right)=\text { Weyl } \\
& \Longleftrightarrow T-\mu_{1} I=\text { Weyl for each } i=1,2, \cdots, n \\
& \Longleftrightarrow \mu_{i} \notin \omega(T) \text { for each } i=1,2, \cdots, n \\
& \Longleftrightarrow \lambda \notin p(\omega(T))
\end{aligned}
$$

which says that $\omega(p(T))=p(\omega(T))$. If $f$ is analytic on a neighborhood of $\sigma(T)$, then there is a sequence $\left(p_{n}\right)$ of polynomials such that $f_{n} \rightarrow f$ uniformly on $\sigma(T)$. Since $p_{n}(T)$ commutes with $f(T)$, by Oberai $[8]$

$$
f(\omega(T))=\lim p_{n}(\omega(T))=\lim \omega\left(p_{n}(T)\right)=\omega(f(T)) .
$$

Recall that $T \in B(H)$ is said to be isoloid if iso $\sigma(T) \subset \pi_{0}(T)$ (Oberai [9]).

LEMMA 2.3. (Oberai [9]) Let $T \in B(H)$ be isoloid. Then for any polynomial $p(t)$, $p\left(\sigma(T)-\pi_{00}(T)\right)=\sigma(p(T))-\pi_{00}(p(T))$.

Let $T$ be an $M$-hyponormal operator which satisfies the additional property that for all $z$ in the complex plane, all integers $n$ and all $x$ in $H$,

$$
\left\|(T-z)^{n} x\right\|^{2}<M\left\|(T-z)^{2 n} x\right\| \cdot\|x\| .
$$

$T$ is said to be an operator of $M$-power class $(N)$ (Istrătescu [7]). The following $M$ - hyponormal operator $T$ which is not hyponormal is of $M$-power class $(N)$ (Istrătescu [7]): Let $\left\{e_{\imath}\right\}$ be an orthonormal basis for $H$, and define

$$
T e_{\imath}=\left\{\begin{array}{l}
e_{2}, \quad \text { if } \quad i=1 \\
2 e_{3}, \quad \text { if } i=2 \\
e_{\imath+1}, \quad \text { if } i \geq 3
\end{array}\right.
$$

i.e., $T$ is a weighted shift. From the definition of $T$ we see that $T$ is similar to the unilateral shift $U$ (Halmos [5], Problem 90). Thus there exists an $S$ such that $T=S U S^{-1}$. In our case $\|S\|=2,\left\|S^{-1}\right\|=1$. Since $U$ is the unilateral shift, $U$ is a hyponormal operator, and thus for every $n$ and $z \in \mathbb{C}$ the operator $(U-z)^{n}$ is of class $(N)$. It follows that

$$
\left\|(U-z)^{n} x\right\|^{2} \leq\left\|(U-z)^{2 n} x\right\|
$$


for all $x \in H$ with $\|x\|=1$, and hence $T$ is of $M$-power class with $M=4$. Thus our class is strictly larger than the class of hyponormal operators. Since $w(T)=w(U)=D$ (the closed unit disc) and $\pi_{0}(T)=\emptyset, \sigma(T)=w(T)$ and so Weyl's theorem holds for $T$.

THEOREM 2.4. If $T \in B(H)$ is an operator of $M$-power class $(N)$, then for any polynomial $p$ on a neighborhood of $\sigma(T)$ Weyl's theorem holds for $p(T)$.

PROOF. By Istrătescu [7], $T$ is isoloid and Weyl's theorem holds for any operator of $M$-power class $(N)$. Hence by Theorem 2.2 and Lemma 2.3 ,

$$
w(p(T))=p(w(T))=p\left(\sigma(T)-\pi_{00}(T)\right)=\sigma(p(T))-\pi_{00}(p(T))
$$

Therefore Weyl's theorem holds for $p(T)$.

Since every hyponormal operator is of 1 -power class $(N)$, we obtain the following result which answers the question of Oberai.

COROLLARY 2.5. If $T \in B(H)$ is hyponormal, then for any polynomial $p$ on a neighborhood of $\sigma(T)$ Weyl's theorem holds for $p(T)$.

ACKNOWLEDGMENT. I wish to express my appreciation to the referee whose remarks and observations lead to an improvement of the paper. This paper was partially supported by Cheju National University Research Fund, 1996

\section{REFERENCES}

[1]. BERBERIAN, S.K., An extension of Weyl's theorem to a class of not necessary normal operators, Michigan Math. J., 16 (1969), 273-279.

[2]. BERBERIAN, S.K., The Weyl's spectrum of an operator, Indiana Univ. Math. J., 20(6) (1970), 529-544..

[3]. CONWAY, J.B., Subnormal operators, Pitman, Boston, 1981.

[4]. GRAMSCH, B. and LAY, D., Spectral mapping theorems for essential spectra, Math. Ann., 192 (1971), 17-32.

[5]. HALMOS, P.R., Hilbert space problem book, Springer-Verlag, New York, 1984.

[6]. HARTE, R.E., Invertibility and singularity for bounded linear operators, Marcel Dekker, New York, 1988.

[7]. ISTRĂTESCU, V.I., Some results on $M$-hyponormal operators, Mathematics Seminar Notes, 6 (1978).

[8]. OBERAI, K.K., On the Weyl spectrum, Mlinois J. Math., 18 (1974), 208-212.

[9]. OBERAI, K.K., On the Weyl spectrum II, Illinois J. Math., 21 (1977), 84-90.

[10].Schechter, M., Principles of functional analysis, Academic Press Inc., New York, 1971.

[11].WADHWA, B.I., M-hyponormal operators, Duke Math. J., 41(3) (1974), 655-660. 


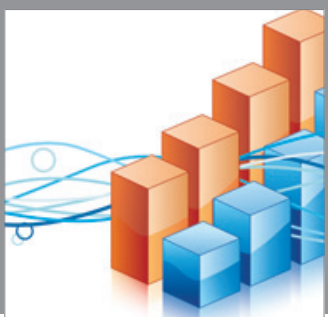

Advances in

Operations Research

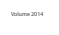

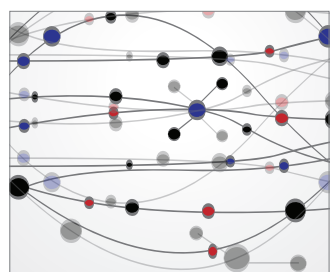

\section{The Scientific} World Journal
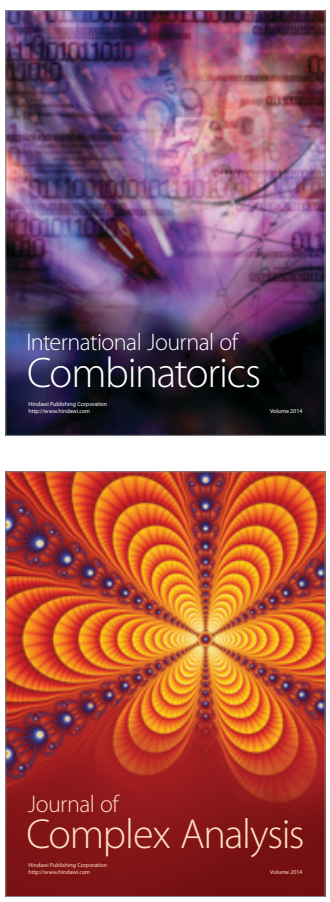

International Journal of

Mathematics and

Mathematical

Sciences
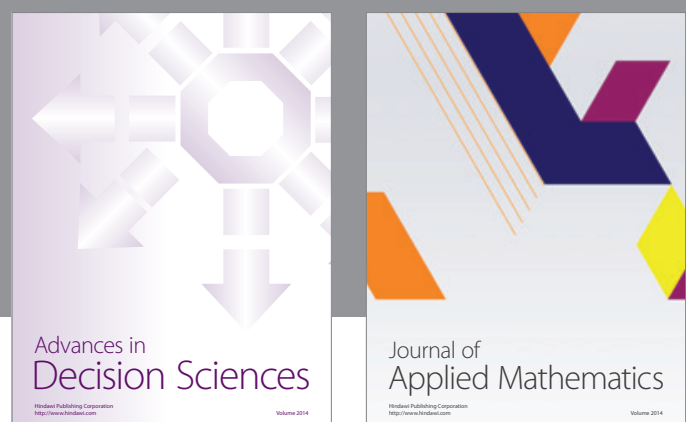

Journal of

Applied Mathematics
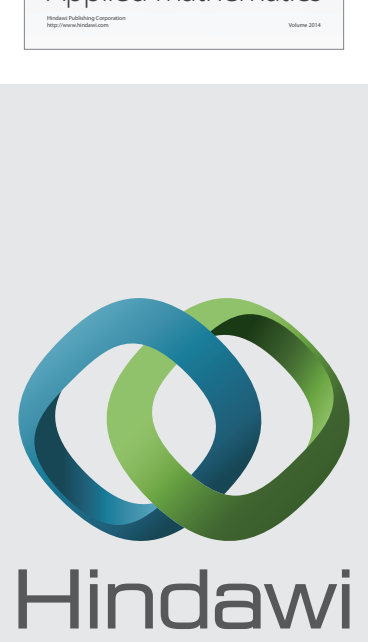

Submit your manuscripts at http://www.hindawi.com
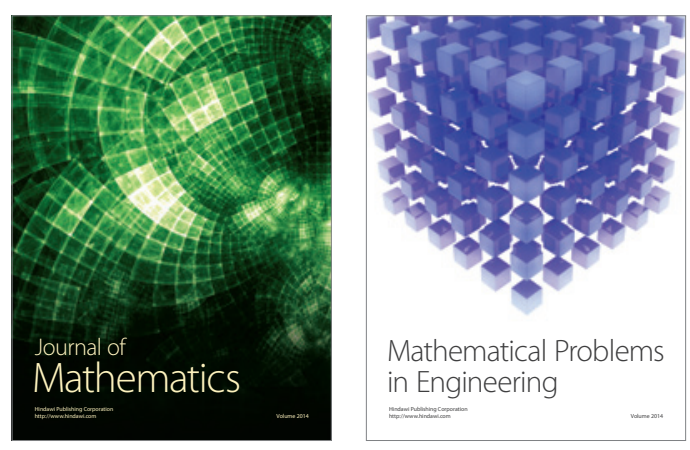

Mathematical Problems in Engineering
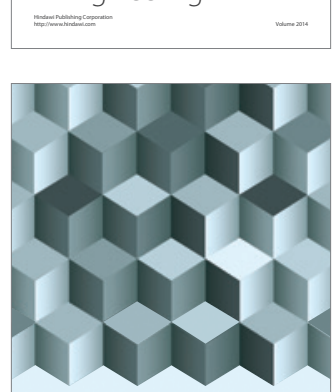

Journal of

Function Spaces
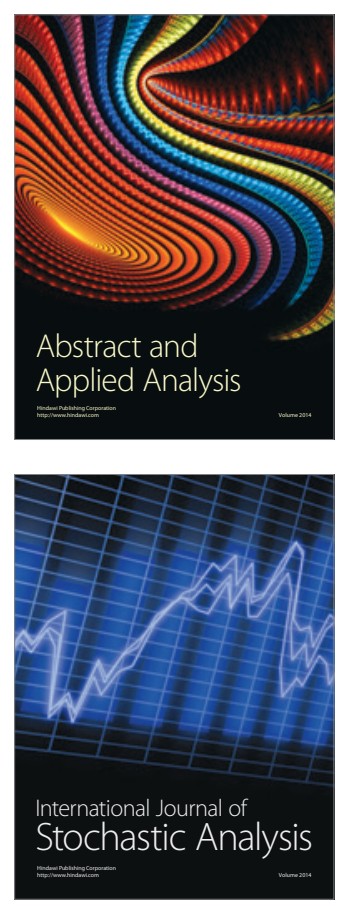

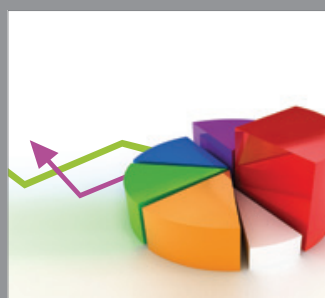

ournal of

Probability and Statistics

Promensencen
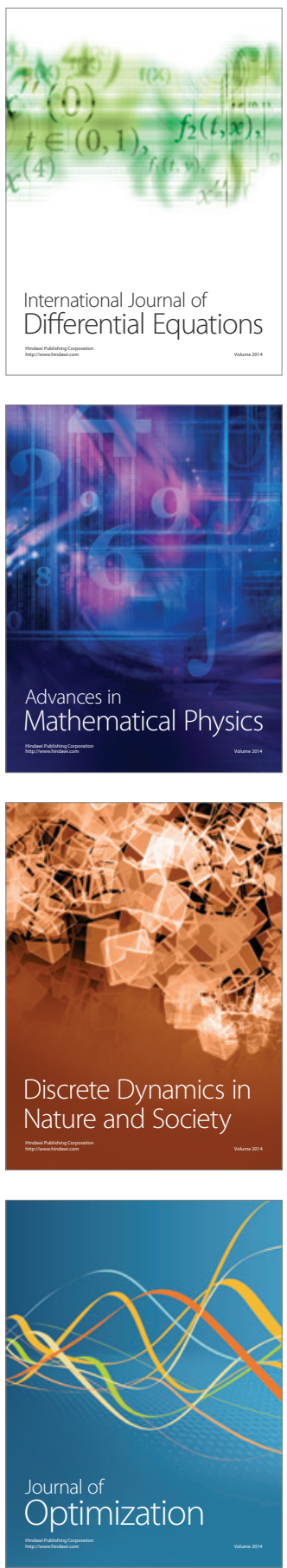